\title{
Emergence of nutraceuticals as the alternative medications for pharmaceuticals
}

\begin{abstract}
Nutraceuticals are the food or a part of it that have some health beneficial effects with or without nutritional values. For past several decades, these nutraceuticals have been gaining importance as the alternative or supplemental medications along with pharmaceuticals for prevention or treatment of wide-range of diseases. These nutraceuticals have been an attractive option over the conventional therapies due to their less or no side effects and nutritional values. Although the effectiveness of nutraceuticals as complementary medications has proven in several Pre-/clinical trials, a significant scientific debate is going on their medical benefits. So, there is an emerging need to demonstrate the health benefits (in alone or combination) of nutraceuticals and provide their detailed molecular mode of action. In this mini review, I have attempted to summarize the beneficial effects and uses of several nutraceuticals (dietary supplements, phytochemicals, nutrients, and herbals) against some of the chronic ailments including cancer, diabetes, obesity, cardiovascular, and neurological diseases.
\end{abstract}

Keywords: nutraceuticals, health benefits, therapeutics, cancer, diabetes, cardiovascular disease, obesity, neurological disease, phytochemicals
Volume II Issue 3 - 2018

\author{
Upendarrao Golla ${ }^{1,2}$ \\ 'Department of Biological Sciences, Indian Institute of Science \\ Education and Research Bhopal (IISERB), India \\ ${ }^{2}$ Department of Pediatrics, Penn State College of Medicine, USA
}

Correspondence: Upendarrao Golla, Department of Pediatrics, Penn State College of Medicine, Hershey, PA I7033, USA, Email uug2!@psu.edu, gollaupendarrao@gmail.com

Received: March 23, 2018 | Published: May 23, 2018

\section{Introduction}

The term 'Nutraceutical' was first devised in 1989 by Stephen Defelice, and it is defined as "any substance that is a food or a part of the food and provides medical or health benefits, including the prevention and treatment of disease". ${ }^{1}$ These nutraceuticals including the isolated nutrients, dietary supplements (minerals, vitamins, amino acids, and herbs), specific diets to genetically engineered designer foods, and phytochemicals (flavonoids, phytosterols, polyphenols, tannins) promised to have beneficial health effects besides the traditional nutrition values found in food. There is a significant association between the dietary habit and the determination of chronic diseases such as cataract, cardiovascular diseases, diabetes, gallstones, inflammatory diseases, neurodegenerative diseases (Alzheimer's/Parkinson's/Huntington's disease), obesity, and several types of cancers. ${ }^{2,3}$ So, the nutraceuticals have been used to improve health and increase the life expectancy, delay the aging process and prevent chronic diseases, or support the structure and/or function of the body. The major benefits of nutraceuticals over pharmaceuticals include lesser or no adverse effects, economically affordable, easily available, multiple therapeutic effects, and increase the health value by improving medical condition of humans. Recently, the nutraceuticals have received substantial attention due to their potential nutritional values, safety, and therapeutic effects. Accordingly, the recent analysis by $\mathrm{BCC}$ research proposed that the global nutraceutical market is expanding at a compound annual growth rate (CAGR) of $7.5 \%$, would reach US $\$ 285.0$ billion by 2021 from US $\$ 198.7$ billion in $2016 .{ }^{4}$ This short review highlights the emerging scope of nutraceuticals as alternative medications for the health management and treatment of several chronic diseases.

\section{Pharmacological implication of nutraceuticals}

\section{Nutraceuticals in obesity}

Obesity is one of the global health problems and a common chronic disease manifested clinically with the excessive accumulation of body fat that might have negative effects on the health. According to the recent WHO report in 2014, the worldwide incidence and prevalence of obesity have been increasing rapidly with $39 \%$ of adults $(38 \%$ of men and $40 \%$ of women) aged $18 \mathrm{yrs}$ and over were overweight, and $13 \%$ were obese. ${ }^{5}$ The occurrence of obesity and overweight happens due to energy imbalance between calories intake (food consumption), and calories expended (biochemical and metabolic processes).The excessive fat accumulation in obesity leads to metabolic changes that increase the morbidity risks and predisposes the individual to the development of many non-communicable diseases such as cancer, congestive heart failure, diabetes, hyperlipidemia, hypertension, respiratory disorders, osteoarthritis, and reduced fertility [6]. Therefore, rationale for the choice of healthier foods, nutraceutical interventions, and exercise are being investigated as potential components in prevention and treatment of obesity. ${ }^{7}$

An ideal nutraceutical for the management of obesity should be able to increase energy expenditure and/or decrease caloric intake for body weight reduction. Several marketed nutraceuticals such as Amla (Emblica officinalis), Curcumin, Fenugreek (Trigonella foenumgraecum), and vitamins (Chromium, Magnesium, and vitamin E) were proven to be effective against obesity and its associated metabolic complications. ${ }^{8}$ The other nutraceuticals including capsaicin conjugated linoleic acid, Murraya koenigii, Momordica charantia, Lagenaria siceraria, Vigna mungo, and Psyllium fiber possess potential anti-obese properties along with hypoglycemic and hypolipidemic effects. ${ }^{8}, 9$ Moreover, the herbal stimulants namely caffeine, chitosan, ephedrine, green tea (Camellia sinensis), ma huang-guarana can be useful in facilitating the body weight loss. ${ }^{10}$

\section{Nutraceuticals in cardiovascular diseases}

Heart diseases account majorly for the deaths worldwide, and the prevalence of cardiovascular disease (CVD) is increasing due to several risk factors including lifestyle (diet, smoking, physical activity) and 
metabolic conditions (diabetes, hypertension, hyperlipidemia, and obesity). In general, CVD is used to describe the disorders related to heart and blood vessels, and includes the peripheral vascular diseases, coronary heart disease (heart attack), cerebrovascular disease (stroke), heart failure, hypertension, etc. It was supposed that low intake of fruits and vegetables associated with high mortality in CVD. The majority of CVDs are preventable, and several studies have reported a beneficial role for a diet rich in fruits and vegetables against CVD. ${ }^{11}$

The nutraceutical molecules including dietary fibers, vitamins, polyphenols, minerals, flavonoids (flavones/flavanones/flavonols), antioxidants, and polyunsaturated fatty acids (PUFAs) are believed to play a beneficial role in alone and/or together with physical exercise for the prevention and curing of CVDs. ${ }^{12}$ The intake of food such as vegetables, cruciferous, grapefruits, nuts, endives, apples, pomegranate, cherries, berries, onion, Buckwheat seeds that are rich in flavonoids and dietary fibers is inversely associated with the incidence and mortality from CVDs. ${ }^{13,14}$ In addition, the phytosterols (stigmasterol, beta-sitosterol, campesterol), anthocyanins, stilbenes, tannins, fatty acids of the omega-3 series ( $n-3$ fatty acids), octacosanol, serotonin, melatonin, and hesperidin in plant foods have potential protective effects in prevention and alleviation of heart diseases including CVDs and myocardial infarction. ${ }^{15,16}$ As reviewed by Zuchi $\mathrm{C}$ et al. ${ }^{17}$ several nutraceuticals exhibit beneficial effects in CVDs through modulating the endothelial functions. ${ }^{17}$ Hence, the nutraceuticals will have major implications in the treatment of CVDs with less/no side-effects.

\section{Nutraceuticals in diabetes}

Diabetes mellitus is one of the chronic metabolic disorders where the carbohydrate utilization is impaired in the body due to absolute or relative lack of insulin hormone that is produced in the pancreas by $\beta$-islets of Langerhans. Commonly, type 2 diabetes (insulin resistance) is the widespread disease with $95 \%$ prevalence, expected to affect $4.4 \%$ of the world population by 2030 and impose a considerable economic burden on society. ${ }^{18}$ Type 2 diabetes is associated with obesity due to increased insulin resistance caused by the adipocyte-derived hormone-like compounds. ${ }^{19}$ Also, it increases the cardiovascular risks and causes diabetic neuropathy, nephropathy, and diabetic retinopathy. Although there are various therapeutic interventions including diet, exercise, and drugs available for the clinical management and treatment of diabetes, epidemiological increase in the number of people with diabetes is reflecting its multifactorial nature.

In recent years, several nutraceuticals and herbal dietary supplements used in clinical practice have been scientifically proven to benefit type 2 diabetes and their complications by targeting the pathogenesis. ${ }^{20}$ These compounds include $\alpha$-lipoic acid, omega-3 fatty acids, berberine, dietary fibers, phytoestrogens (Isoflavones), antioxidant vitamins (vitamins $\mathrm{C}$ and $\mathrm{E}$ ), vitamin $\mathrm{D}$, flavonoids, conjugated linoleic acid, and minerals (chromium and magnesium. ${ }^{21,22}$ The dietary fibers from psyllium have been shown to aid in weight reduction and control glucose levels in diabetic patients. ${ }^{23}$ The $\alpha$-lipoic acid, a universal antioxidant that is used for the treatment of diabetic neuropathy can be an effective dietary supplement for prevention of diabetic complications. Also, cinnamon tea, green tea, and ethyl esters of $n-3$ fatty acids can be helpful for the management of diabetic complications. ${ }^{24}$ The consumption of Soy isoflavones and Omega-3 fatty acids have been shown to decrease glucose tolerance in diabetes and associated with lower incidence and mortality rate of heart disease, osteoporosis, and certain cancers. ${ }^{25}$

\section{Nutraceuticals in cancer}

Cancer is one of the emerging global health issues with the constant increase in its incidence and mortality. Cancer can be described as an uncontrolled proliferation of abnormal cells that invade into surrounding tissue. According to the World Cancer Report, the cancer rates are rising, and it would reach 15 million new cases by the year 2020 that is, a rise in $50 \%$. The rapidly proliferating cancer cells undergo several adaptations in the metabolism of lipids, fat, carbohydrate, and proteins to meet the cellular demands. As per the reports from several well-controlled clinical trials, a healthy lifestyle with the inclusion of diet rich in nutraceuticals/antioxidants can help in the prevention of cancer and improve the overall quality of life. ${ }^{26}$ The nutraceuticals with antioxidant property have been shown to decrease the oxidative DNA damage and modulate several cellular signaling pathways such as EGFR, COX-2, PI3K/Akt, NF- $\mathrm{KB}$, and AMPK-mTOR to decrease the cancer aggressiveness and thus provide the beneficial effects. ${ }^{27}$

Several phytochemicals that are antioxidant in nature including carotenoids, flavonoids from citrus fruit, polyphenolics compounds like epigallocatechin, gallate, tocopherol, curcumin, genistein, and $\beta$-carotene exhibit chemopreventive properties by acting as antioxidants. ${ }^{28}$ The foods rich in cysteine, lycopene (tomatoes, guava, pink grapefruit, watermelon, papaya), glutathione, Vitamin E, Vitamin C, soy, ellagic acid (walnuts, berries, pomegranates, and pecans) and selenium was shown to elevate the total antioxidative capacity. However, further detailed studies are needed to test their protective effects in cancer prevention and/or treatment. ${ }^{29}$ The polyphenol Curcumin from Curcuma longa possesses antioxidant, anticancer and anti-inflammatory properties. Thus, several clinical trials are underway to evaluate curcumin and its derivatives against several types of cancers as an individual and/or in combination with other chemotherapeutics. ${ }^{30}$ Also, the saponins (found in peas, tomatoes, alfalfa, potatoes, spinach, soybeans, and clover) reported to exhibit anticancer and antimutagenic activities, thus lowers the risk of cancers. Tannins that are found in grapes, tea, lentils, and berries are potent antioxidants and potential anticancer molecules that quench the harmful free-radicals and thus prevent the oxidative damage to DNA. ${ }^{31}$

Nowadays, the demand for dietary foods and phytochemicals with beneficial effects in cancer-prevention is high and gained more attention. Although large-scale clinical trials have shown the beneficial role of phytochemicals and nutraceuticals as an adjuvant to the conventional chemotherapy or radiotherapy, we need more elaborative studies to define the value of alternative and complementary medicine in combating cancers.

\section{Nutraceuticals in Nurodegenerative diseases (NDDs)}

The neurodegenerative diseases (NDDs) including Alzheimer's, amyotrophic lateral sclerosis (ALS), Huntington's, and Parkinson's are incurable and debilitating conditions that arise as a result of progressive neuronal degeneration (loss of structure or function of neurons) including the death of nerve cells in the human brain. These NDDs mostly cause complications with movement (also called ataxias), or mental functioning (also called dementias), and behavioral problems including depression..$^{32}$ The NDDs show similar characteristics including the increased occurrence with age (>50-60) and are mostly sporadic in nature, although less-prevalent familial can occur with early onset. These NDDs have a higher prevalence and become a global health problem due to demographic changes and lack 
of effective treatments. Although the etiology of neurodegeneration remains elusive, there are recent evidences indicating that the damage to neuronal mitochondria (mitotoxicity), increased intracellular calcium $\left(\mathrm{Ca}^{2+}\right)$ levels, oxidative stress, and persistent inflammatory conditions in alone or combination of any of these are well-recognized mechanisms leading to neurotoxicity. ${ }^{33}$ Moreover, the epidemiological studies revealed the role of several genetic and environmental factors along with the age as significant risk factors for the initiation and progression of neurodegeneration and thus NDDs.

The diversity and complexity of cell death mechanisms in neurodegeneration and adverse effects associated with the chronic use of current therapeutic agents promoted the use of nutraceuticals or herbal medicines as safety and commercial approaches for the prevention/ treatment of NDDs. Additionally, the ability of nutraceuticals to concurrently act on multiple pathways linked to neuronal cell death resulted in the growing interest of their usage to improve the clinical manifestations associated with NDDs. ${ }^{34}$ To name a few, the emerging nutraceuticals such as curcumin, $\alpha$-lipoic acid, astaxanthin, coenzyme Q10 (ubiquinone), L-sulforaphane (isothiocyanate Compound), tert-butyl hydroquinone, blue berry, resveratrol, carnosic acid, eugenol, emodin (3-methyl-1,6,8trihydroxyanthraquinone), rosmarinic acid, aged garlic extract, anthocyanins, epigallocatechin-3-gallate (green tea flavonoid), mustard oil glycoside, retinoic acid, Vitamin D, Vitamin E, Omega-3 polyunsaturated fatty acid, apigenin, Soy isoflavones, and isoflavones were shown to have beneficial effects in NDDs by modulating one or more abovementioned mechanisms of neurodegeneration (reviewed in). ${ }^{33}$ The nutraceutical antioxidants including astaxanthin, curcumin, $\beta$-carotene, $\alpha$-lipoic acid, quercetin, lutein, lycopene, coenzyme Q10 (ubiquinone), resveratrol, and turmerin have been shown to combat oxidative stress-mediated diseases and thus exhibit protective effects against aging, dementia, and NDDs. ${ }^{35}$

\section{Conclusion}

In conclusion, it is evident from growing attention and market value that these nutraceuticals will play a critical role in the healthcare system as medications in alone or combined with modern therapeutics. The safety (less adverse effects), cost-effectiveness, and nutritional values with the use of nutraceuticals make them as an attractive, reliable, and suitable option for prevention or treatment of diseases. The higher use of nutraceuticals these days also warns us to have a regulatory authority to control the quality and avoid their misusage. In these modern days, the emerging technologies should be implicated to prove the clinical effectiveness along with their detailed mode of action, and thus support the evidence-based usage of nutraceuticals as alternative and complementary medications to provide the ultimate health benefits to mankind. Finally, I am sure that the nutraceutical industry will flourish in the near future and stand as an excellent option for providing the therapeutic benefits.

\section{Acknowledgement}

None.

\section{Conflict of interest}

Author declares there is no conflict of interest towards this manuscript.

\section{References}

1. Defelice SL. The Nutraceutical Revolution - Its Impact on FoodIndustry R-and-D. Trends Food Sci Tech. 1995;6(2):59-61.

2. Smith-Warner SA, Elmer PJ, Tharp TM, et al. Increasing vegetable and fruit intake: Randomized intervention and monitoring in an at-risk population. Cancer Epidem Biomar Prev. 2000;9(3):307-317.

3. Andersen V, Holst R, Vogel U. Systematic review: diet-gene interactions and the risk of colorectal cancer. Aliment Pharm Ther. 2013;37(4):383391.

4. https://www.bccresearch.com/market-research/food-and-beverage/ nutraceuticals-markets-report-fod013f.html

5. World Health Organization (WHO). Obesity and overweight in 2016. Geneva: World Health Organization; 2016.

6. Caterson ID, Gill TP. Obesity: epidemiology and possible prevention. Best Pract Res Clin Endocrinol Metab. 2002;16(4):595-610.

7. Das L, Bhaumik E, Raychaudhuri U, et al. Role of nutraceuticals in human health. J Food Sci Tech Mys. 2012;49(2):173-183.

8. Kaur G, Mukundan S, Wani V. et al. Nutraceuticals in the Management and Prevention of Metabolic Syndrome. Austin J Pharmacol Ther. 2015;3(1):1063.

9. Rubin SA, Levin ER. Clinical review 53: The endocrinology of vasoactive peptides: synthesis to function. J Clin Endocrinol Metab. 1994;78(1):6-10.

10. Boozer CN, Nasser JA, Heymsfield SB, et al. An herbal supplement containing Ma Huang-Guarana for weight loss: a randomized, doubleblind trial. Int J Obesity. 2001;25(3):316-324.

11. Hu FB, Willett WC. Optimal diets for prevention of coronary heart disease. JAMA. 2002;288(20):2569-78.

12. Shahbazian H. World diabetes day; 2013. J Renal Inj Prev. 2013;2(4):123-4.

13. Khosravi-Boroujeni H, Sarrafzadegan N, Mohammadifard N, et al. White rice consumption and CVD risk factors among Iranian population. J Health Popul Nutr. 2013;31(2):252-61.

14. Li SQ, Zhang QH. Advances in the development of functional foods from buckwheat. Crit Rev Food Sci Nutr. 2001;41(6):451-64.

15. Garg A, Garg S, Zaneveld LJ. Chemistry and pharmacology of the Citrus bioflavonoid hesperidin. Phytother Res. 2001;15(8):655-69.

16. Sidhu KS. Health benefits and potential risks related to consumption of fish or fish oil. Regulatory Regul Toxicol Pharmacol. 2003;38(3):33644.

17. Zuchi C, Ambrosio G, Lüscher TF, et al. Nutraceuticals in cardiovascular prevention: lessons from studies on endothelial function. Cardiovasc Ther. 2010;28(4):187-201.

18. King H, Aubert RE, Herman WH. Global burden of diabetes, 1995 2025: prevalence, numerical estimates, and projections. Diabetes Care. 1998;21(9):1414-31.

19. Jackson MB, Ahima RS. Neuroendocrine and metabolic effects of adipocyte-derived hormones. Clin Sci (Lond). 2006;110(2):143-52.

20. Rafieian-Kopaei M, Nasri H. Ginger and diabetic nephropathy. J Renal Inj Prev. 2013;2(1):9-10.

21. Davì G, Santilli F, Patrono C. Nutraceuticals in diabetes and metabolic syndrome. Cardiovasc Ther. 2010;28(4):216-26. 
22. Derosa G, Limas CP, Macías PC, et al. Dietary and nutraceutical approach to type 2 diabetes. Arch Med Sci. 10(2):336-44.

23. Singh B. Psyllium as therapeutic and drug delivery agent. Int J Pharm 2007;334(1-2):1-14.

24. Sirtori CR, Galli C. N-3 fatty acids and diabetes. Biomedicine \& pharmacotherapy. 2002;56(8):397-406.

25. Tavafi M. Diabetic nephropathy and antioxidants. J Nephropathol. 2013;2(1):20-7.

26. Roudebush P, Davenport DJ, Novotny BJ. The use of nutraceuticals in cancer therapy. The Veterinary clinics of North America Small animal practice. 2004;34(1):249-69.

27. Li Y, Go VL, Sarkar FH. The Role of Nutraceuticals in Pancreatic Cance Prevention and Therapy: Targeting Cellular Signaling, MicroRNAs, and Epigenome. Pancreas. 2015;44(1):1-10.

28. Thomasset SC, Berry DP, Garcea G, et al. Dietary polyphenolic phytochemicals - promising cancer chemopreventive agents in humans? A review of their clinical properties. Int J Cancer. 2007;120(3):451-8.
29. Ardalan MR, Rafieian-Kopaei M. Antioxidant supplementation in hypertension. J Renal Inj Prev. 2014;3(2):39-40.

30. Amalraj A, Pius A, Gopi S, et al. Biological activities of curcuminoids, other biomolecules from turmeric and their derivatives - A review. $J$ Tradit Complement Med. 2016;7(2):205-233.

31. Li H, Wang Z, Liu Y. Review in the studies on tannins activity of cancer prevention and anticancer Zhong Yao Cai. 2003;26(6):444-8.

32. Przedborski S, Vila M, Jackson-Lewis V. Neurodegeneration: what is it and where are we? J Clin Invest. 2003;111(1):3-10.

33. Dadhania VP, Trivedi PP, Vikram A, et al. Nutraceuticals against Neurodegeneration: A Mechanistic Insight. Curr Neuropharmacol. 2016;14(6):627-40.

34. Ong WY, Sun GY, Wood WG, et al. Nutraceuticals in Neurodegeneration and Aging. Neuromolecular Med. 2016;18(3):239-40.

35. Kelsey NA, Wilkins HM, Linseman DA. Nutraceutical antioxidants as novel neuroprotective agents. Molecules. 2010;15(11):7792-814. 